\title{
ASA, NSAIDs, coxibs and colorectal cancer prevention - How far have we come?
}

\author{
Martin Fishman MD FRCPC
}

\begin{abstract}
ARTICLE
Rahme E, Barkun AN, Toubouti Y, Bardou M. The cyclooxygenase-2-selective inhibitors rofecoxib and celecoxib prevent colorectal neoplasia occurrence and recurrence. Gastroenterology 2003;125:404-12.
\end{abstract}

\begin{abstract}
ARTICLE SUMMARY
This paper described a nested case-control study on all Quebec patients over 65 years of age who underwent total colonic imaging or surgery for colorectal cancer (CRC) during a specified six-month period without similar testing in the preceding year. Information from the provincial government database was collected for each subject to estimate the consumption of prescription acetylsalicylic acid (ASA), standard nonsteroidal anti-inflammatory drugs (NSAIDs) and cyclooxygenase-2specific inhibitors (coxibs) during the previous year. Of 2568 subjects, 730 were found to have at least one adenoma (but not carcinoma) and 129 were found to have CRC (with or without adenoma). Patients with and without adenoma or carcinoma were compared for type, amount and duration of drug exposure to determine if there was a protective effect. Rofecoxib and NSAIDs were associated with reduced incidence of colorectal adenoma (CRA), and these drugs and celecoxib protected against CRA and CRC.
\end{abstract}

\section{DISCUSSION}

CRC prevention is increasingly prominent as a public health issue and as a reason for referral to gastroenterologists. Because of our awareness of the adenoma-carcinoma sequence and evidence that adenoma detection by screening reduces CRC incidence and mortality, screening has emerged as the pre-eminent modality of CRC prevention. The resources invested in CRC screening are large and increasing, and have been the subject of a great deal of economic analysis. Indeed, according to many published models, the cost effectiveness of CRC screening compares favourably with other preventive health measures.

Less public attention has been paid to other CRC prevention strategies, but there is increasing interest in dietary and pharmacological approaches. Public awareness that supplements like selenium and vitamin D may reduce CRC risk has declared itself in our offices and has probably been of benefit to the burgeoning 'nutriceutical' industry. There is also emerging interest in the potential role of ASA, long the easy friend of cardiologists but the nemesis of gastroenterologists. Are we about to start seeing ASA and its more upscale offshoots, the NSAIDs and coxibs, in a less inflammatory way?

The paper reviewed here outlines the background and rationale for using these drugs in CRC prevention in the general population. Cox-2 expression is vastly greater in neoplastic tissue than in normal colorectal mucosa. Cox-2 inhibition results in CRC prevention and in CRA prevention and regression in animals, as has been previously reviewed in this Journal and elsewhere (1-5). Similar effects have been noted in patients with familial adenomatous polyposis, in whom the role of coxibs is chiefly to retard the development and progression of intestinal neoplasms for which surgery may be deemed inappropriate (6-9).

The role of Cox-2 inhibition in the chemoprevention of sporadic CRC is less well established. Early studies suggested a benefit from ASA, and this has again been demonstrated recently (10-13). Other NSAIDs have also recently been shown to decrease the prevalence of CRC or advanced CRA (14). The study under review here aimed to measure and compare any preventive effects of ASA, standard NSAIDs, rofecoxib and celecoxib on sporadic CRA and CRC.

In this study, rofecoxib exposure protected against CRA, with an odds ratio (OR) of 0.67. The effect was enhanced (OR 0.37) by taking high dosages for at least three months. For celecoxib, a difference from controls (OR 0.76) could be demonstrated only for exposure to high dosages for at least three months. Standard NSAIDs also were protective against CRA (OR 0.41).

For CRC prevention, celecoxib was most effective (OR 0.23), followed by rofecoxib (OR 0.53) and standard NSAIDs (OR 0.67). When data for CRA and CRC were combined, the ORs were $0.47,0.64$ and 0.73 for standard NSAIDs, rofecoxib and celecoxib, respectively. For patients with previous colorectal neoplasia, only rofecoxib was protective (OR 0.43). Insufficient numbers of patients took high dosages 
of ASA or NSAIDs for at least three months to ascertain either a dose or duration effect.

This was the first study examining the potential role for coxibs in preventing sporadic CRA and CRC. Given the greater gastrointestinal safety of these agents compared with ASA and standard NSAIDs, these agents may prove to be the drugs of choice, depending on the results of future prospective studies. Cost will clearly need to be factored into any models that examine this question. Risk stratification will also likely be crucial, and will need to consider those most likely to benefit from coxibs (possibly those with a previous personal or family history of sporadic CRA or CRC) and those most likely to be harmed. The story on ASA for CRC prevention is even less well worked out, and its use even for cardioprotection is likely best reserved for selected patients because of its risks.

An additional observation made in this study is worthy of comment. The likelihood of finding CRA or CRC was smaller for patients with bowel symptoms, other than bleeding, than for asymptomatic patients, who were presumably investigated for screening purposes rather than for diagnosis. This finding supports the emerging opinion of many experts that, for the assessment of symptoms like chronic abdominal pain or constipation, colonoscopy should not be considered essential, and the choice of investigation should be determined by the individual clinical situation.

This study has some shortcomings. The authors acknowledge that over-the-counter use of ASA and ibuprofen by subjects was not identified or estimated. Furthermore, it is not known whether anticipated use of the drugs might have prompted anticipatory investigation for gastrointestinal bleeding, which may have resulted in detection of CRA or CRC before the drug was prescribed. Conversely, blood loss resulting from use of these drugs might have provoked testing that led to the discovery of CRA or CRC. Finally, two limitations common to this field of study also apply here, namely that the study was not prospective, and that the duration of drug exposure and follow-up might have been insufficient to fully assess the long-term benefit of these drugs.

This paper sets the stage for longer term prospective studies. Based on the potential revenue coxibs could generate if proven to prevent cancer, their manufacturers may be able to justify the funding of such trials. Subject recruitment may be largely comprised of patients somewhat younger than those examined in this case-control study, but it is easy to imagine that a multicentre collaboration would be able to enroll sufficient numbers to provide adequate statistical power for such a prospective study. Until then, most of us will still view this class of drugs with cautious respect.

\section{REFERENCES}

1. Arber N. Do NSAIDs prevent colorectal cancer? Can J Gastroenterol 2000;14:299-307.

2. Turner D, Berkel HJ. Nonsteroidal anti-inflammatory drugs for the prevention of colon cancer. CMAJ 1993;149:595-602.

3. Thun MJ. NSAID use and decreased risk of gastrointestinal cancers. Gastroenterol Clin North Am 1996;25:333-48.

4. Thun MJ. Aspirin and gastrointestinal cancer. Adv Exp Med Biol 1997;400A:395-402.

5. Lancaster T, Silagy C. Aspirin and neoplasia of the digestive tract: Is there a chemopreventive effect? Dig Dis 1994;12:170-6.

6. Giardiello FM, Hamilton SR, Krush AJ, et al. Treatment of colonic and rectal adenomas with sulindac in familial adenomatous polyposis. N Engl J Med 1993;328:1313-6.

7. Rigau J, Pique JM, Rubio E, Planas R, Tarrech JM, Bordas JM. Effects of long-term sulindac therapy on colonic polyposis. Ann Intern Med 1991;115:952-4

8. Labayle D, Fischer D, Vielh P, et al. Sulindac causes regression of rectal polyps in familial adenomatous polyposis. Gastroenterol 1991;101:635-9.

9. Waddell WR, Ganser GF, Cerise EJ, Loughry RW. Sulindac for polyposis of the colon. Am J Surg 1989;157:175-9.

10. Suh O, Mettlin C, Petrelli NJ. Aspirin use, cancer and polyps of the large bowel. Cancer 1993;72:1171-7.

11. Greenberg ER, Baron JA, Freeman DH Jr, Mandel JS, Haile R. Reduced risk of large-bowel adenomas among aspirin users. The Polyp Prevention Study Group. J Natl Cancer Inst 1993;85:912-6.

12. Sandler RS, Halabi S, Baron JA, et al. A randomized trial of aspirin to prevent colorectal adenomas in patients with previous colorectal cancer. N Engl J Med 2003;348:883-90.

13. Baron JA, Cole BF, Sandler RS, et al. A randomized trial of aspirin to prevent colorectal adenomas. N Engl J Med 2003;348:891-9.

14. Lieberman DA, Prindiville S, Weiss DG, Willett W, and the VA Cooperative Study Group 380. Risk factors for advanced colonic neoplasia and hyperplastic polyps in asymptomatic individuals. JAMA 2003;290:2959-67.

\section{The authors respond:}

We appreciate Dr Fishman's thoughtful comments. The readers will note that the three-month period of drug intake is essentially used as a proxy, in such studies, for more prolonged use of the specified medications. Additionally, any over-thecounter intake of NSAIDs or ASA would indeed not be captured by the provincial government database, even though recent data suggest that such use is minimal in patients over age 65 (the target group for this analysis). Nevertheless, the use of these drugs would only mean that some of the patients classified as nonexposed were in fact exposed to the drugs of interest and would thus have tended to diminish any observable protective effect that could be attributed to the intake of non-selective NSAIDs or coxibs. It seems unlikely that the anticipated use of these drugs might have prompted anticipatory investigation for GI bleeding; indeed screening for lower GI tract lesions prior the start of any NSAID or coxib treatment has not been recommended.

Drs Elham Rahme, Alan Barkun and Marc Bardou 


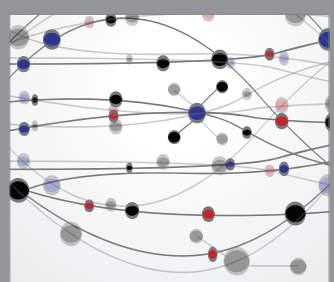

The Scientific World Journal
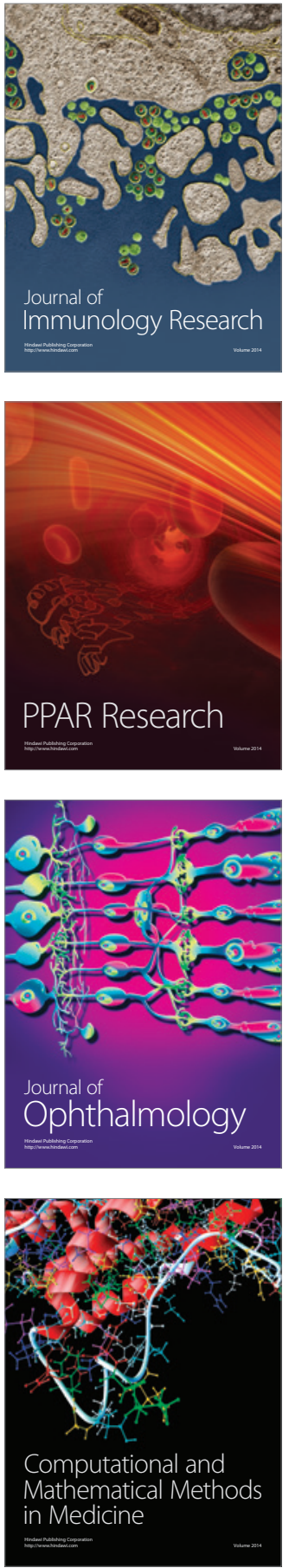

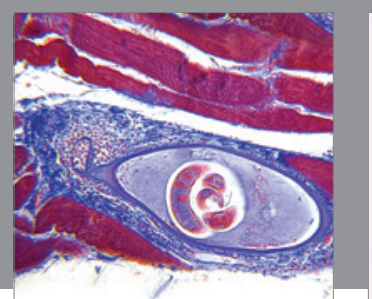

Gastroenterology Research and Practice

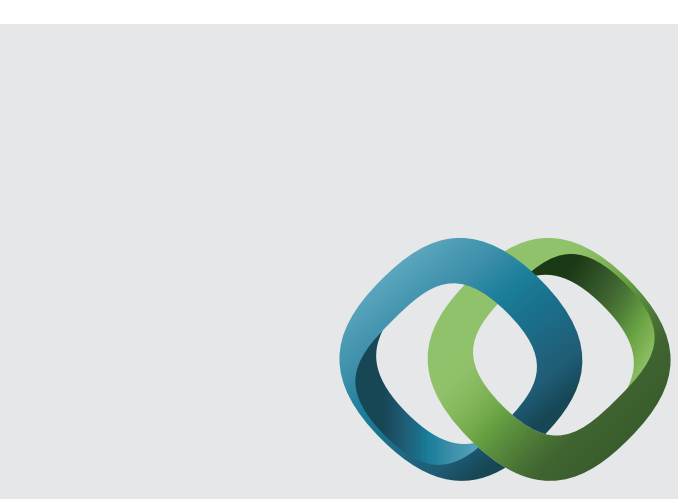

\section{Hindawi}

Submit your manuscripts at

http://www.hindawi.com
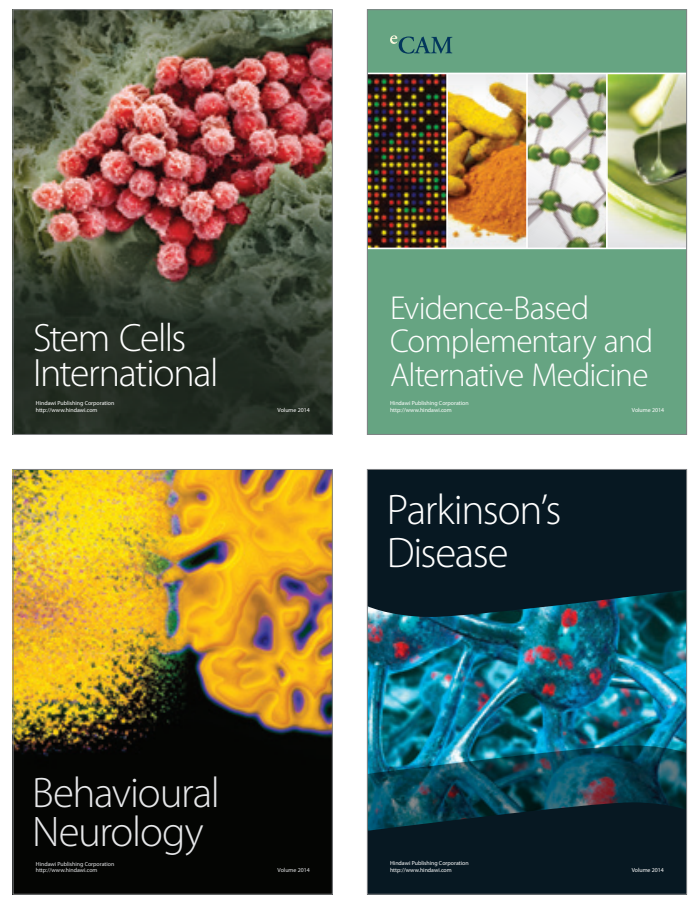
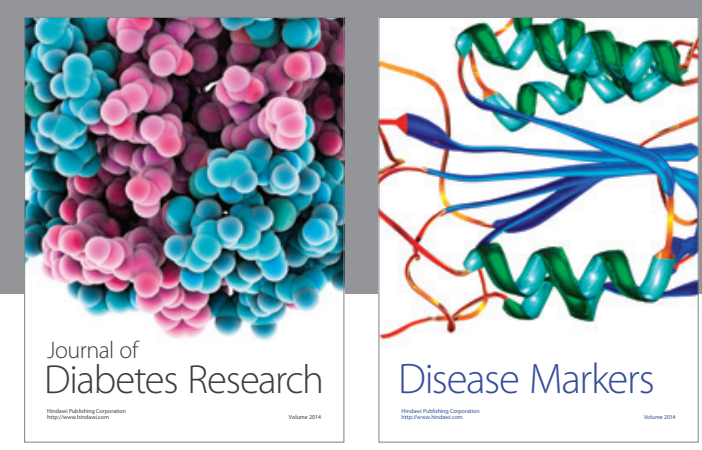

Disease Markers
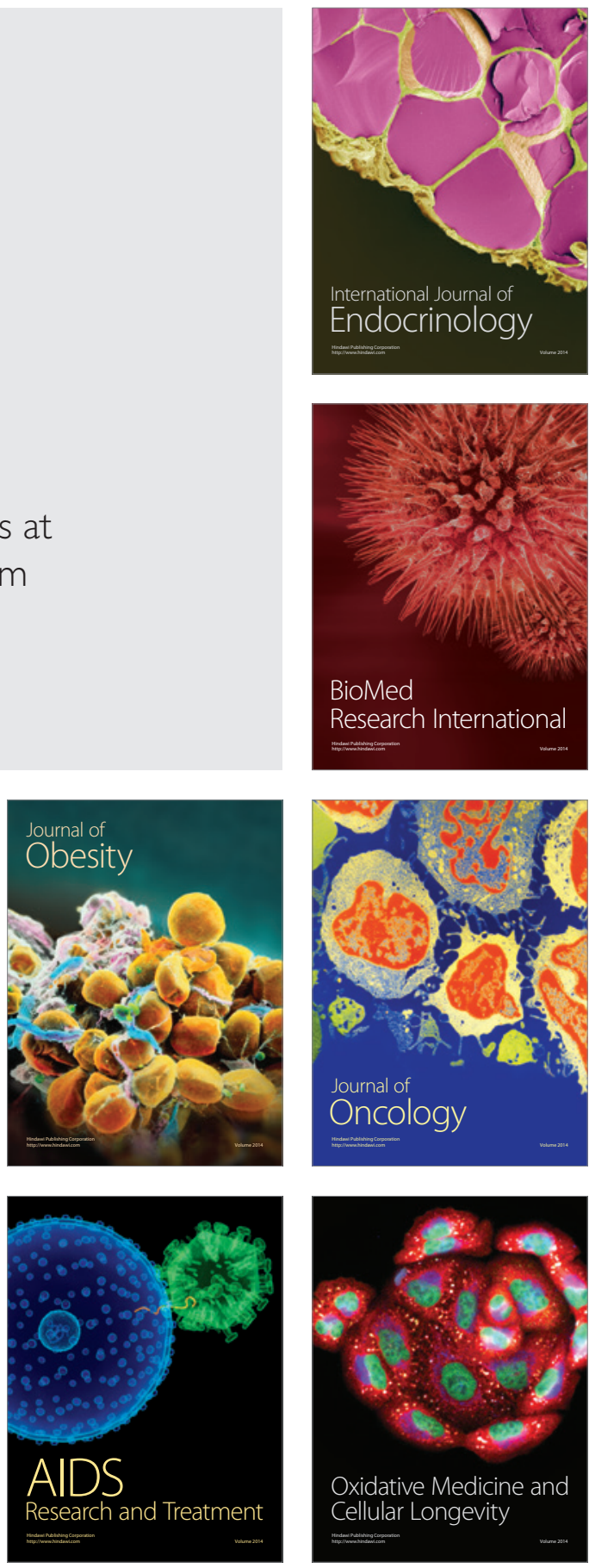